\title{
Numerical Investigation of a Process Control for the Roller Levelling Process based on a Force Measurement
}

\author{
Markus Grüber ${ }^{1, a^{*}}$ and Gerhard Hirt ${ }^{1, b}$ \\ ${ }^{1}$ Institute of Metal Forming (IBF), RWTH Aachen University, Intzestr. 10, 52056 Aachen, Germany \\ agrueber@ibf.rwth-aachen.de, ${ }^{b}$ hirt@ibf.rwth-aachen.de
}

Keywords: Process control, Finite element method (FEM), Roller levelling.

\begin{abstract}
When processing conventional semi-finished metal strips, distinctive changes in the material properties along the strip length are unavoidable. The roller levelling process is sensitive to changes of those strip characteristics. Thus, a process control allowing for an online adaption of the roller levelling machine according to the actual strip characteristics is highly desirable. In order to enable a precise process layout, the calculation by the Finite Element Method (FEM) provides a suited strategy. Furthermore, the coupling of user-subroutines to an FE code offers the possibility to implement and test respective control strategies. This work proposes a control strategy that is based on a force measurement in the first load triangle of a levelling machine. A first FE model including a feedback control is used to calculate the dependence between the force in the first load triangle and the roll intermesh in the last load triangle leading to a flat sheet. The results are transferred to meta models - so called control curves - that give a direct relationship between the measured force and the roll intermesh. Within a second FE setup a feed-forward control based on these control curves is implemented and the proposed control strategy is investigated for varying yield strengths along the strip length. Thus, the time consuming FE simulations that are necessary to obtain the control curves are decoupled from the actual levelling process. According to the obtained results, the introduced approach is able to improve the sheet flatness for thin sheets when a change in the material properties occurs.
\end{abstract}

\section{Introduction}

When feeding sheet metals through conventional sheet processing lines, a crucial step is to pitch the decoiled strip into the first step of the processing line. Commonly, roller levelling machines are used to ensure a certain flatness and thereby a stable guidance of the strip to the actual processing line [1]. The roller levelling process is basically a reversed bending operation the metal strip undergoes when passing through the machine. Each single bending is defined by the positions of the rolls with respect to each other. These roll positions are defined by the so called roll intermesh. The intermesh refers to the roll position relative to the pitches of the lower rolls. A small value of the roll intermesh leads to greater bending, that is a greater curvature, of the sheet metal (Fig. 1).

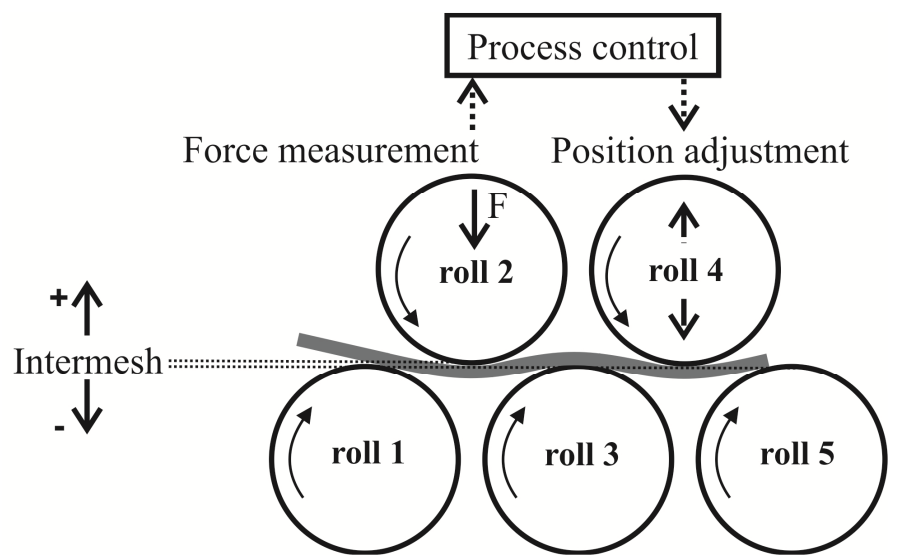

Figure 1: Schematic view of the roller leveller with proposed feed-forward control 
The process of roller levelling itself is sensitive to both geometrical design parameters of the levelling machine as well as the material properties of the sheet metal [2,3]. A widely used possibility of an accurate process layout for the roller levelling process is the use of FE simulations [4,5]. Despite the high accuracy of such FE simulations, the corresponding high computational times of conventional FE codes are a major drawback for an online process control [6]. This work introduces a feed-forward process control that relies on numerically calculated control curves which correlate the calculated bending force in the first load triangle, the respective yield strength of the sheet metal as well as the roll intermesh of the last load triangle resulting in a flat strip (Fig. 1). These control curves offer the possibility of a fast online adaption of the roller levelling machine.

\section{State of the Art}

Due to the high demand for a process control in the application of roller levelling, several works have been conducted with the goal to introduce a suitable process control.

Doege et al. [7] introduced a process control that is based on an analytical model including several simplifications that is capable of calculating the expected forces at each levelling roll. Along the process the forces acting on the rolls are measured and deviations from the calculated forces are compensated by a feedback process control which directly acts on the individual roll positions.

Several works focused on using a measurement of the residual curvature at the machines outlet in experimental setups instead of a force measurement [8,9]. In those experimental setups the curvature measurement is done by measuring the distance of the metal strip to a reference positon by laser-based devices or capacitive measurement techniques respectively. It has been noted by the authors that the influence of gravity as well as the strip guidance on the sheet curvature must not be neglected. In consequence, all of those measurement techniques can only provide representative values but not a quantitative value of the actual curvature. In the case of heavy plate levellers the influence of the guidance can be compensated by coupling several independent measurement devices [10].

Nonetheless, the own weight of the metal strip will always affect the measured curvature. A possible way to overcome these disturbing influences is the use of FE simulations. Within the idealised environment of an FE simulation it is possible to obtain the resulting curvature at the machine's outlet without superimposed disturbances such as gravity.

To determine the roll positions leading to a flat product Pernía et al. [11] introduced an approach employing genetic algorithms for optimising the process parameters. As the optimisation by such genetic algorithms requires a large number of simulation runs, a different approach is the use of feedback control-loops within an FE code. Oligschläger et al. [12] proposed a setup where the residual sheet curvature is estimated by a single position measurement at the machine's outlet. Kaiser et al. [13] presented an FE model that uses pre-calculated curvature values for each single bending operation the investigated long product undergoes when passing through the roller leveller as reference for a control-loop. These FE setups including a control-loop allow for a determination of the optimised roll positions in a single simulation run for a given set of process parameters.

Alternatively, several works propose analytical models with simplifying assumptions that are based on fundamental equations allowing for a fast calculation [14]. The high sensitivity of the roller levelling process makes an accurate positioning of the rolls in the range of micrometres necessary [15]. As analytical models always are a compromise between computational time and accuracy, it is unavoidable to use FE simulations when looking for most accurate results [16].

A look on the afore summarised works in the field of roller levelling shows the strong demand for establishing an online process control. In order to combine both, the high accuracy of FE simulations as well as fast computational times, this work presents a method based on fast meta models. To obtain sampling points for the meta model, an FE setup including a feedback control is used and the results are summarised in so called control curves. Thus, the fast process of roller levelling is separated from the time consuming FE simulations 


\section{Methods}

Determination of Control Curves. In a first step an FE model including a feedback control that has been introduced in [17] is used to calculate the control curves. This FE model employs a plain strain assumption in a 2D setup of a five roll levelling machine. The control curves correlate the force of the first load triangle and the roll intermesh in the last load triangle for different strip characteristics. In general, three different sheet thicknesses of $\mathrm{s}=1.5 \mathrm{~mm}, \mathrm{~s}=2.0 \mathrm{~mm}$ and $\mathrm{s}=2.5 \mathrm{~mm}$ are investigated. For each of these thicknesses the initial flow stress has been increased in equidistant intervals of $10 \mathrm{MPa}$ starting from the initial flow stress $\sigma_{\mathrm{Y}}$ up to $\sigma_{\mathrm{Y}}+60 \mathrm{MPa}$. By increasing the initial flow stress in this manner, typical variations of the strip characteristics as they may occur during a levelling process are reflected. As testing material the mild steel DC01 is used and to obtain representing material parameters quasi-static tensile tests have been performed. The results are depicted in Fig. 2 and the characteristic values of the Young's modulus as well as the initial flow stress $\sigma_{\mathrm{Y}}$ are given in Table 1.

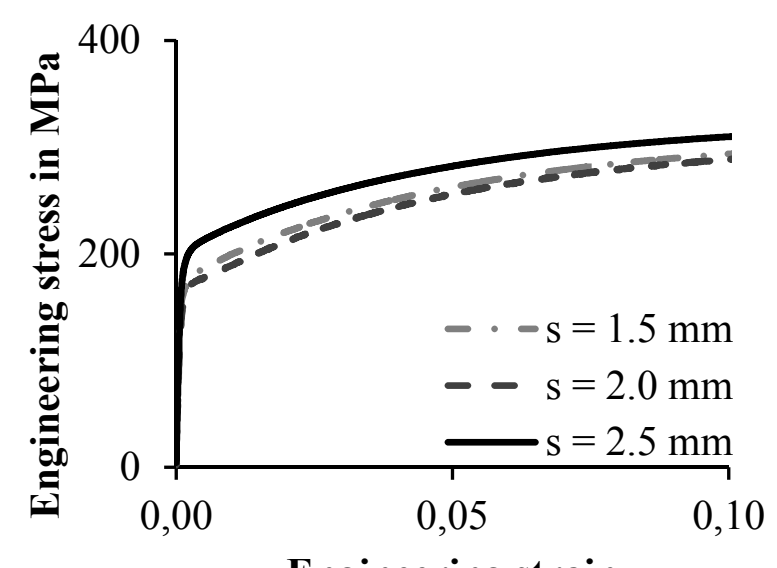

Engineering strain

a)

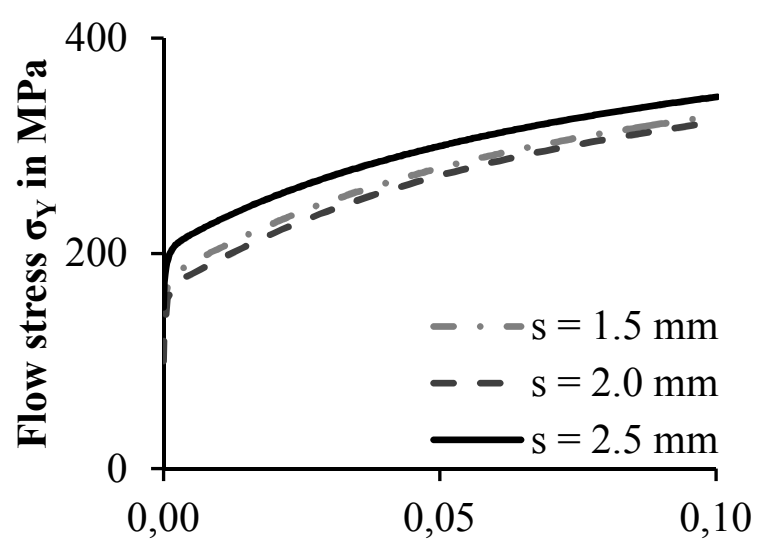

True strain

b)

Figure 2: Results from tensile tests (a) and flow curves (b) for different sheet thicknesses s

Table 1: Young's modulus E and initial flow stress $\sigma_{\mathrm{Y}}$

\begin{tabular}{|c|c|c|c|}
\cline { 2 - 4 } \multicolumn{1}{c|}{} & $\mathbf{S = 1 . 5} \mathbf{~ m m}$ & $\mathbf{S}=\mathbf{2 . 0} \mathbf{~ m m}$ & $\mathbf{s}=\mathbf{2 . 5} \mathbf{~ m m}$ \\
\hline Young's modulus E in MPa & 189,482 & 200,817 & 207,058 \\
\hline Initial flow stress $\boldsymbol{\sigma}_{\mathbf{Y}}$ in MPa & 150 & 99 & 151 \\
\hline
\end{tabular}

According to the described variation of the initial yield strength $\sigma_{Y}$ for each sheet thickness seven simulation runs are conducted. For each sheet thickness the intermesh of the first load triangle is set to a position leading to a defined plastic strain across the sheet section. Thus, the sheet inherits a defined curvature independent from possible pre-curvatures. The intermesh of the last load triangle is adjusted by a feedback control. If a flat sheet leaves the machine, the corresponding force in the first load triangle and the intermesh in the last load triangle are sampled for the control curves.

Feed-forward Control Algorithm. In the first FE-model the control curves have been determined by using a feedback control. In order to develop and test a feed-forward control algorithm that acts when changes in the strip characteristics occur, a respective control algorithm is implemented to a second FE model. Thus, it is possible to investigate suitable control algorithms for the roller levelling process prior to an implementation to an experimental setup.

The proposed feed-forward control measures the force in the first load triangle continuously with a frequency of $100 \mathrm{~Hz}$. As the force measurement shows a certain signal noise it is necessary to set a threshold value that defines whether a change of the force is due to a change of the material properties or due to signal noise. To distinguish between signal noise and an actual change of the material properties, the mean value of last 15 force sample values is compared to the actual measured force. If the difference between this mean value and the actual value becomes greater than the threshold value, the feed-forward control calculates the intermesh of the last load triangle that 
corresponds to the measured force in the first load triangle. The calculated intermesh of the last load triangle is then compared to the actual intermesh. Based on the difference between calculated intermesh and actual intermesh the feed-forward control sets a velocity for the adjustment of the intermesh in the last load triangle. In general, the feed-forward control algorithm acts as long as a significant change of the force in the first load triangle occurs and the difference between calculated and actual intermesh in the last load triangle is unequal to zero.

\section{Results}

Control Curves. In a first step, the numerical model including a feedback control has been used to determine control curves that give a correlation between the intermesh of roll 4 leading to a flat sheet and the specific force at roll 2. As the numerical model is set in a $2 \mathrm{D}$ environment, the force calculated equals a force value acting on a certain sheet width and thus is given as a specific force per $\mathrm{mm}$ of the sheet width. Fig. 3 shows the respective control curves that have been obtained for three different sheet thicknesses $\mathrm{s}$.
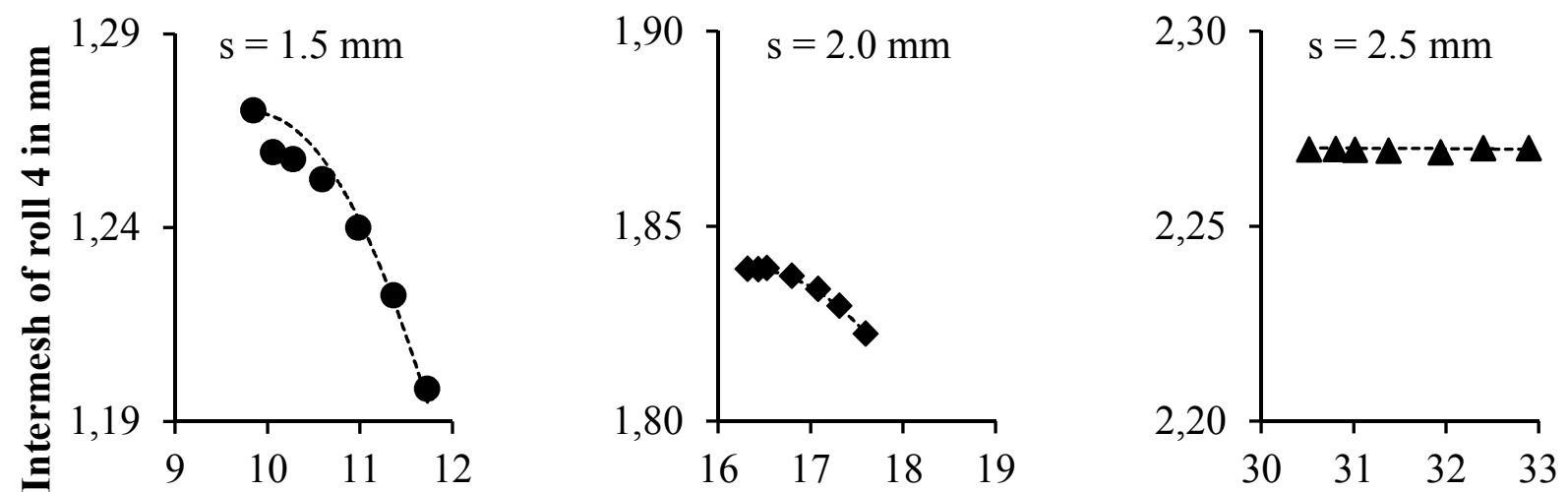

Specific force at roll 2 in $\mathrm{N} / \mathrm{mm}$

Figure 3: Obtained control curves for different sheet thicknesses $\mathrm{s}$

For a sheet thickness of $\mathrm{s}=1.5 \mathrm{~mm}$ and a sheet thickness of $\mathrm{s}=2.0 \mathrm{~mm}$ the intermesh leading to a flat sheet at roll 4 decreases with an increasing force in the first load triangle measured at roll 2. In case of a sheet thickness of $\mathrm{s}=2.5 \mathrm{~mm}$ the change of the intermesh in the last load triangle is negligible. Generally, the increasing force acting on roll 2 is the result of the increasing initial flow stress that has been set in the simulation runs. In consequence, the intermesh at roll 4 decreases with an increasing initial flow stress of the sheet metal. This is due to the fact that a greater value of the initial flow stress leads to a greater total elastic strain during bending. Therefore, a greater bending - or a lower value of the roll intermesh respectively - needs to be imposed by the rolls to achieve a certain plastic strain.

As stated before it is obvious that the required intermesh shows a greater dependency to the force and the initial flow stress respectively for lower sheet thicknesses. This becomes obvious when looking on the range of the intermesh changes for the investigated flow stress variations. Therefore, a process control adapting the intermesh has a greater potential for improving the flatness for thin sheets.

Feed-forward process control. The implemented subroutine adapts the intermesh of roll 4 with respect to the measured force at roll 2 according to the afore described control curves. The results that have been achieved when using this process control are compared to FE simulations without a control in Fig. 4. The initial flow stress of the sheet is increased in steps of $30 \mathrm{MPa}$ every $300 \mathrm{~mm}$ of the sheet length starting from the initial flow stress obtained in the tensile tests. The initial intermesh of roll 4 is set to a position leading to flat sheet for the first initial flow stress. Therefore, an increasing curvature is to be expected when the feed-forward process control is disabled.

The depicted results match with the results of the control curves. It is visible that a low sheet thickness of $1.5 \mathrm{~mm}$ shows a significant increase in the sheet curvature of approx. $60 \cdot 10^{-5} \mathrm{~mm}^{-1}$ 
when the feed-forward control is not enabled. For the same sheet thickness and enabled feedforward control the curvature increases only about $1.7 \cdot 10^{-5} \mathrm{~mm}^{-1}$ and is close to zero over the whole sheet length. When looking on the higher sheet thicknesses of $2.0 \mathrm{~mm}$ and $2.5 \mathrm{~mm}$ it is obvious that - according to the control curves - the influence of a change of the initial flow stress is much smaller and in case of a sheet thickness of $2.5 \mathrm{~mm}$ the influence is negligible. The observed greater curvatures for parts of the sheets with a thickness of $2 \mathrm{~mm}$ and $2.5 \mathrm{~mm}$ with control compared to the cases without control are in range of the accuracy of the numerical curvature measurement and thus do not reflect any tendencies achieved by the control.

The results clearly show that the suggested feed-forward control is capable of improving the flatness after levelling when a change in the initial flow stress occurs along the sheet length. In particular for low sheet thicknesses the process control can lead to a significant improvement.
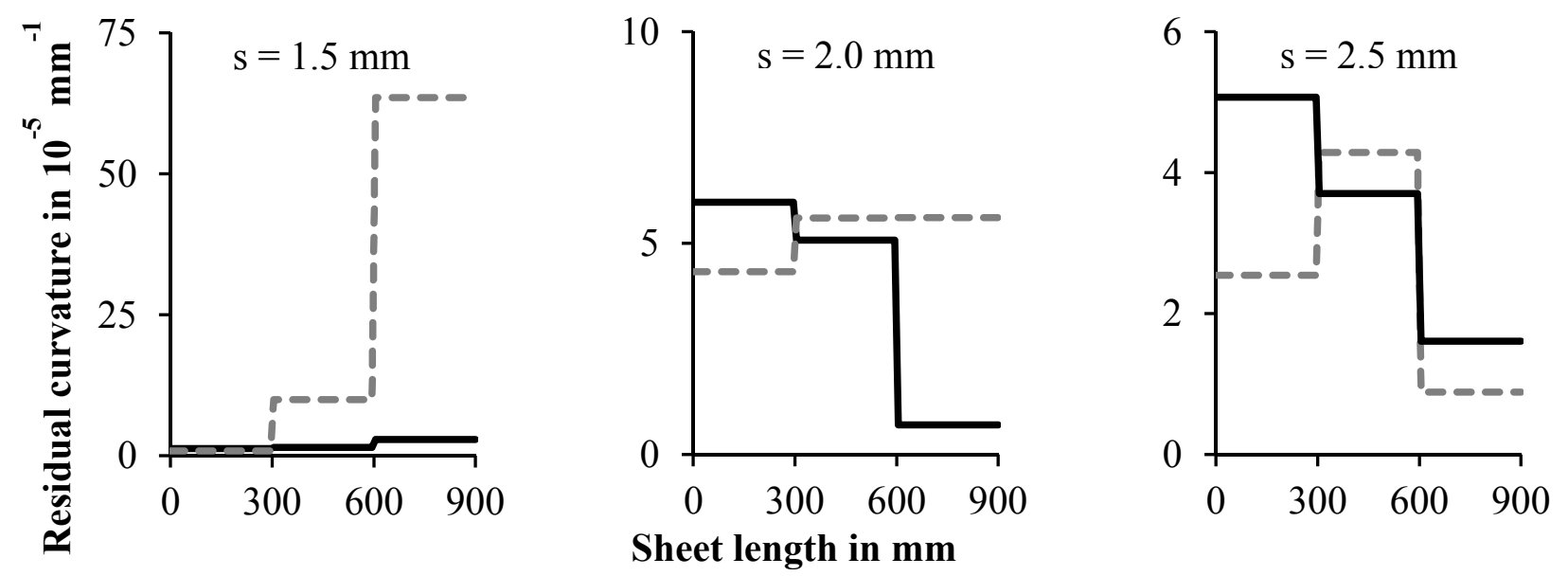

---- without feed-forward control with feed forward control

Figure 4: $\quad$ Flatness with and without feed-forward control for different sheet thicknesses s

\section{Conclusion}

Within this work a feed-forward process control for the roller levelling process is proposed that is capable of adjusting the leveller machine if changes of the flow stress occur along the sheet length. In order to obtain the necessary control curves that correlate the force in the first load triangle of a five roll leveller and the intermesh leading to a flat sheet in the last load triangle, an FE setup including a feedback control is used. The introduced feed-forward process control is based on this control curves and acts due to changes of the measured force in the first load triangle. To investigate the potential of the proposed feed-forward control, the developed control algorithm is implemented to a commercial FE code using a user-subroutine. These investigations have been conducted for three different sheet thicknesses of the mild steel DC01 with varying initial flow stresses.

The results show a clear dependence between the measured force at the first upper roll and the intermesh of the last upper roll in case of lower sheet thicknesses. When comparing the different investigated sheet thicknesses, it is obvious that the influence of changes in the flow stress becomes more prominent for sheet thicknesses below $2.5 \mathrm{~mm}$. When enabling the feed-forward process control, an improvement of the sheet flatness has been achieved, in particular for the low sheet thicknesses.

In conclusion, the proposed strategy of calculating control curves employing a numerical model including a feedback control and using these control curves as basis of a feed-forward control algorithm is a promising way to improve the flatness along the roller levelling process. The implementation to a commercial FE code enables the investigation and enhancement of the proposed feed-forward control prior to transferring the control to an experimental setup. 


\section{Acknowledgements}

The authors would like to gratefully acknowledge the funding provided by the "Deutsche Forschungsgemeinschaft" (DFG) within the research project "Untersuchungen zur Prozessführung des Richtwalzens unter Nutzung eines FE-Modells mit integrierten Regelstrukturen”.

\section{References}

[1] J.W. Morris, S.J. Hardy, J.T. Thomas, Some fundamental considerations for the control of residual flatness in tension levelling, JMPT 120 (2002) 385-396.

[2] H. Huh, J.H. Heo, H.W. Lee, Optimization of a roller levelling process for A17001T9 pipes with finite element analysis and Taguchi method, Int. J. of Mach. Tools \& Man. 43 (2003) 345-350.

[3] J.W. Morris, S.J. Hardy, J.T. Thomas, Effects of tension levelling process parameters on cold rolled strip characteristics using a designed factorial analysis approach, Ironmaking and Steelmaking 32/5 (2005) 443-448.

[4] H. Huh, H.W. Lee, S.R. Park, G.Y. Kim, S.H. Nam, The parametric process design of tension levelling with an elasto-plastic finite element method, JMPT 113 (2001) 714-719.

[5] L. Steinwender, A. Kainz, K. Krimpelstätter, K. Zeman, Numerical Simulation of Tension Losses and Reaction Forces in Tension Levellers, SRI, Special Edition (2011) 343-348.

[6] F. Yoshida, M. Urabe, Computer-aided process design for the tension levelling of metallic strips, JMPT 89-90 (1999) 218-223.

[7] E. Doege, R. Menz, S. Huinink, Analysis of the levelling process based upon an analytic forming model, CIRP Annals - Manufacturing Technology 51/1 (2002) 191-194.

[8] B.-A. Behrens, R. Krimm, Controlled Sheet Metal Straightening, Supplemental Proceedings 3, TMS (2008) 129-136.

[9] R. Haberland, G. Lauer, A sensor system for the controlled roller levelling machine for sheet metal, Blech Rohre Profile 40/7 (1993) 599-601.

[10]L. Bodini, O. Ehrich, M. Krauhausen, Improvements at heavy plate leveller by coupling a model to a flatness gauge, Stahl und Eisen 128/6 (2008) 47-50.

[11]A. Pernía, F.J. Martínez-de-Pisón. J. Ordieres, F. Alba, J. Blanco, Fine tuning straightening process using genetic algorithms and finite element methods, Ironmaking and Steelmaking 37/2 (2010) 119-125.

[12] M. Oligschläger, G. Hirt, Implementation of Closed-loop Control Systems in Finite Element Simulations of Roller Leveling, SF2M Con. Proc. (2012) 43-44.

[13]R. Kaiser, T. Hatzenbichler, B. Buchmayr, T. Antretter, Simulation of the roller straightening process with respect to residual stresses and the curvature trend, Materials Science Forum 768-769 (2014) 456-463.

[14] Y. Cho, H. Ye, S. Hwang, A New Model for the Prediction of Evolution of the Residual Stresses in Tension Leveling, ISIJ International 53/8 (2013) 1436-1442.

[15]B.-A. Behrens, T. El Nadi, R. Krimm, Development of an analytical 3D-simulation model of the levelling process, JMPT 211 (2011) 1060-1068.

[16]B. Dratz, V. Nalewajk, J. Bikard, Y. Chastel, Testing and modelling the behaviour of steel sheets for roll levelling applications, International Journal of Material Forming 2/1 (2009) 519-522.

[17]M. Grüber, M. Oligschläger, G. Hirt, Adjusting of Roller Levellers by Finite Element Simulations Including a Closed-loop Control, Advanced Materials Research 1018 (2014) 207-214. 